\section{Abstract A1}

Histopathological findings in an adult Down syndrome patient with progressive muscle weakness and intellectual deficiency

Bastien Paré, Nicolas Dupré, Peter Gould, François Gros-Louis

Hôpital de l'Enfant-Jésus - CHU de Québec, Québec, Canada

doi:10.1017/cjn.2015.367

We present the case of a 49-year-old male diagnosed with trisomy 21 at an early age, who developed severe intellectual deficiency and progressive muscle weakness of the upper limbs at the age of 47 .

He also complained of pain in the shoulders, and became unable to move his right arm within months. The symptoms worsened over the following year. He progressively stopped walking, developed dysphagia, further impairment of the lower limbs and eventually died.

At the autopsy, the patient presented characteristic craniofacial morphological features of Down syndrome. Macroscopic examination of the brain showed atrophy of the superior temporal gyrus and the frontoparietal cortex, a small hippocampus and dilatation of the lateral ventricles.

Microscopic examination of the brain showed typical features of Alzheimer's disease with amyloid deposits in the cerebral cortex, basal ganglia and cerebellar cortex. Gallyas staining showed the presence of numerous neuritic plaques and widespread neurofibrillary degeneration at the level of the hippocampus. Immunohistological stains for alpha synuclein did not reveal the presence of Lewy bodies.

The spinal cord examination showed atrophy of the corticospinal tract. CD68 immunohistochemistry revealed abundant macrophages in the medullary pyramids and lateral columns and an associated microglial reaction. TDP-43 immunohistochemistry showed a filamentous staining in the cytoplasm and a loss of nuclear staining within motor-neurons. Ubiquitin immunohistochemistry showed weak staining of some spinal nerve roots.

A connection between Alzheimer's disease and Down syndrome has been described extensively in the literature. The higher risk for Alzheimer's disease in people with Down syndrome has been attributed to increased production of amyloid beta due to an extra copy of chromosome 21 , but other genes on chromosome 21 may also play a role, such as superoxide dismutase (SOD1). Little is known about the consequences of trisomy 21 for other neurodegenerative diseases. The present case shows that neurodegenerative disease in Down syndrome patients can take other forms besides Alzheimer's disease, including amyotrophic lateral sclerosis.

CONFLicts OF INTEREST:

None.

\section{Abstract A2}

\section{Panencephalopathic Creutzfeldt-Jakob disease}

Gerard H. Jansen ${ }^{1,2,4}$, Stephen Yip ${ }^{3}$, Alain B. Tremblay ${ }^{4}$, David A. Ramsay ${ }^{5}$

${ }^{1}$ University of Ottawa; ${ }^{2}$ Public Health Agency of Canada; ${ }^{3}$ University of British Columbia; ${ }^{4}$ Eastern Ontario Regional Laboratory Association; ${ }^{5}$ London Health Science Centre doi:10.1017/cjn.2015.368
Creutzfeldt-Jakob disease is a rare, fatal neurodegenerative disease, with an average annual mortality of all sporadic, genetic, and infectious acquired forms together in Canada is 1.47 per million per year (last 5 years).

While mostly gross brain abnormalities in CJD are minimal, and white matter abnormalities are hardly ever seen, panencephalopathic CJD (pCJD) is a very rare form of CJD where there is substantial atrophy with brain weights below 1,000 gram, and where white matter pathology is easily found.

Over the course of only 2 years 5 cases of pCJD were recognized within the Canadian CJD surveillance.

Currently there have been 47 cases of pCJD reported in literature. pCJD cases with a sporadic, genetic and iatrogenic background have all been described. Many of the patients in literature are of Japanese background, but not all. The only significant differences between these cases and other CJD cases are the relatively long disease duration (mean 24.5 month, compared to 6 month in all CJD cases) and the morphological findings. As yet there is no explanation for the occurrence of these cases in general, nor for this time-cluster in Canada.

These cases reinforce the message that MRI findings of severe brain atrophy and/or white matter pathology in patients with rapidly progressive neurological disease do not exclude CJD as diagnosis.

CONFLICTS OF INTEREST:

None.

\section{Abstract A3}

Familial dystonia with cerebral calcification: case report and genetic update

M. Signaevski ${ }^{1,2,3}$, Z.K. Wszolek ${ }^{1,2,3}$, A.J. Stoessel ${ }^{1,2,3}$, R. Rademakers ${ }^{1,2,3}$, I.R. Mackenzie ${ }^{1,2,3}$

${ }^{1}$ Vancouver General Hospital, British Columbia, Canada; ${ }^{2}$ Mayo Clinic Jacksonville, Florida, USA; ${ }^{3}$ Pacific Parkinson Research Centre, British Columbia, Canada

doi:10.1017/cjn.2015.369

We present a 71-year-old woman who suffered from dysarthria and a progressive movement disorder with prominent dystonia, on-setting at age 10. CT scans during adulthood demonstrated extensive symmetric calcification of the basal ganglia, thalamus, cerebellum, cerebral white matter and cortex. Biochemical studies, including serum calcium, phosphate and iron levels were normal. Towards the end of her life, she experienced depression, but remained mentally intact. Many other members of her large Canadian family suffered from a similar dystonia-plus syndrome associated with cerebral calcification.

Post mortem examination demonstrated extensive calcification of the brain parenchyma and blood vessels, ranging from small calcospherites to large solid concretions. Recently, whole-genome sequencing identified a $\sim 563 \mathrm{~kb}$ genomic deletion on chromosome 8 affecting multiple genes, including SLC2OA2 and TAHP1, that segregated with disease in the family. SLC2OA2 encodes a type III sodium-dependent phosphate transporter and loss-offunction mutations were recently identified as an important cause of familial and sporadic idiopathic basal ganglia calcification that 